\title{
A chromospheric scenario for the activity of $\beta$ Pictoris, as revealed by FUSE
}

\author{
J.-C. Bouret ${ }^{1}$, M. Deleuil ${ }^{1,2}$, T. Lanz ${ }^{3,4}$, A. Roberge ${ }^{5}$, A. Lecavelier des Etangs ${ }^{6}$, and A. Vidal-Madjar ${ }^{6}$ \\ ${ }^{1}$ Laboratoire d'Astrophysique de Marseille, Traverse du Siphon, BP 8, 13376 Marseille Cedex 12, France \\ 2 Université de Provence, Centre de Mathématiques et d'Informatique, 39 rue J. Curie, 13453 Marseille Cedex 13, France \\ e-mail: Magali.Deleuil@astrsp-mrs.fr \\ 3 Laboratory for Astronomy and Solar Physics, Code 681, NASA Goddard Space Flight Center, Greenbelt MD 20771, USA \\ 4 Department of Astronomy, University of Maryland, College Park, MD 20742, USA \\ e-mail: lanz@stars.gsfc.nasa.gov \\ 5 Department of Physics and Astronomy, Johns Hopkins University, 3400 N. Charles St., Baltimore MD 21218, USA \\ e-mail: akir@pha.jhu.edu \\ ${ }^{6}$ Institut d'Astrophysique de Paris, CNRS, 98bis Bd Arago, 75014 Paris, France \\ e-mail: lecaveli@iap.fr \\ e-mail: alfred@iap.fr
}

Received 5 March 2002 / Accepted 22 April 2002

\begin{abstract}
We investigate a chromospheric scenario to explain the characteristics of the far-UV emission lines of $\beta$ Pictoris, revealed by FUSE spectra of this famous circumstellar disk system. The model assumes a thin region heated up to a few $10^{5} \mathrm{~K}$ located close to the stellar photosphere. The resonance lines of C III at $977 \AA$ and O VI at 1032-1037 $\AA$, seen in emission, are produced in this chromosphere-transition region complex. Using complementary data in the mid and near UV (specifically, a resonance doublet of C IV at 1548-1550 $\AA$ and Mg II h \& k), we show that the whole dataset is remarquably well reproduced by the model. In addition, we investigate the properties of the $\mathrm{C}$ III* multiplet at $1176 \AA$ and conclude that this line likely forms in a weak warm wind, originating from the prominent circumstellar disk of $\beta$ Pictoris. Finally, radiative losses have been calculated and have provided estimates of the amount of non radiative energy dissipated in the external atmosphere of $\beta$ Pictoris, which is a measure of the star's activity level. Such behavior for an A5 V star presents a challenge for both evolution and activity models since the former predict that main-sequence A stars should not be active, while the latter are unable to quantitatively account for the characteristics of the chromospheric heating of $\beta$ Pictoris.
\end{abstract}

Key words. line: profiles - stars: atmospheres - stars: chromospheres - stars: activity - stars: winds, outflows

\section{Introduction}

Recent studies have confirmed that $\beta$ Pictoris is a $20 \pm$ 10 Myr old A5 star, arriving at the main-sequence (Barrado y Navascues et al. 1999). This star has been long known to host a massive debris disk of dust and gas, seen nearly edge-on (Smith $\&$ Terrile 1984). Spectroscopic observations have shown that many ionization states are present in the circumstellar (CS) gas associated with the disk, and they produce numerous variable absorption features, interpreted in terms of star-grazing comets in the Falling Evaporating Bodies (hereafter FEBs) scenario of Lagrange et al. (1988).

Besides derivation of the fundamental stellar parameters, the star itself attracted little interest for further studies, as main-sequence A-type stars like $\beta$ Pictoris are not expected to be chromospherically active. Indeed, according to the standard theory of stellar evolution, main-sequence mid A-type

Send offprint requests to: J.-C. Bouret,

e-mail: Jean-Claude.Bouret@astrsp-mrs.fr stars with intermediate masses, possess at the very best, very thin outer convection zones unable to sustain magnetic activity. Moreover, chromospheric emission lines had never been detected in the spectroscopic data accumulated for years, supporting the conclusion that $\beta$ Pictoris is not an active star. In this context, the recent detection of strong emission lines of O VI and C III with the Far Ultraviolet Spectroscopic Explorer (FUSE) by Deleuil et al. (2001) is very surprising.

Only collisional processes can produce these ions, the stellar FUV of $\beta$ Pictoris being much too weak to ionize a significant fraction of atoms into such high ionization states. This implies that densities and temperatures high enough for collisional processes to dominate are reached in the line formation regions. Such conditions, with electron densities ranging from a few $10^{6} \mathrm{~cm}^{-3}$ to about $10^{10} \mathrm{~cm}^{-3}$ are easily obtained in the FEBs framework, through collisions in shock waves surrounding infalling comet-like bodies. They have been invoked to explain the presence of time variable $\mathrm{Al} \mathrm{III} \mathrm{and} \mathrm{C} \mathrm{IV} \mathrm{absorption}$ lines in $\beta$ Pictoris spectrum (Beust \& Tagger 1993). 
However, this model is unable to explain FUSE observations of O VI and C III. First, the large blueshift of the C III* $\lambda 1175$ line, contrasted with the small redshift of the O VI lines and C III $\lambda 977$, is statistically unlikely, as shown recently by Beust \& Morbidelli (2000). Second, since emission lines arising from highly ionized species are expected to be produced from infalling bodies that are very close to the star, the emission lines should all be redshifted by several hundreds of $\mathrm{km} \mathrm{s}^{-1}$ and should vary rapidly (within hours). FUSE spectra of $\beta$ Pictoris taken at different times (2000 March 18, 2001 March 1, and 2001 March 3 and, more recently, in october 2001) show no significant variation in the emission line fluxes or redshifts, on a timescale of a few days.

On the other hand, it is now observationally well established that several A stars show such an activity. The onset of stellar activity for these objects is however limited to spectral type A7 or later. FUV emission lines from O VI, N V, C III, Si III, C II, and HI have been observed on $\alpha$ Aql (Altair) and $\alpha$ Cep (both of A7 V spectral type) using IUE, HST/GHRS, ORFEUS-SPAS II and FUSE (e.g. Freire Ferrero et al. 1995; Simon \& Landsman 1997; Simon \& Ayres 1998; Gouttebroze et al. 1999; Redfield et al. 2002). These emission lines are observed at similar strengths in the solar spectrum. In the latter case, it is well known that they are produced in the chromosphere and/or in the transition zone between the chromosphere and the corona. Furthermore, chromospheric models have been proposed recently for $\alpha$ Aql and $\alpha$ Cep that successfully account for the shapes and intensities of several emission lines including $\operatorname{Ly} \alpha$ and the C II resonance doublet (Gouttebroze et al. 1999). Non-standard physical mechanisms are now being proposed to solve the problem of the generation of strong magnetic fields that could drive stellar activity in the absence of a significant convective envelope (see e.g. Vigneron et al. 1990; Tout \& Pringle 1995; Lignières et al. 1996).

Such signs of chromospheric and transition region activity were not expected for main-sequence A5 stars, before FUSE observations of $\beta$ Pictoris. In this specific case, the flux levels measured by Deleuil et al. (2001) for the emission lines seem compatible with formation in a solar-like chromospheretransition region (see Table 2). Therefore, we decided to investigate a similar scenario for $\beta$ Pictoris in which the observed emission lines originate in a chromosphere-like region located within a few tenths of stellar radii above the photosphere. Using non-LTE radiative transfer techniques together with semiempirical atmosphere models, line profiles have been computed and compared to observed ones. The atmospheric structure deduced from this modeling has been compared to that of mainsequence A stars of later type as well as to that of the pre-mainsequence Herbig Ae stars, both cases where chromospheric activity is commonly observed.

This allows us to address the question of the evolution with time of the activity of A-type stars, and related to this, the question of the driving mechanisms that sustain the activity.

\section{Selection of the observational material}

The goal of the modeling work presented in this paper is to explain the characteristics of the emission lines of $\beta$ Pictoris observed by FUSE. In addition to C III lines (977 $\AA$ and $1776 \AA$ ) and O VI resonance doublet (1032-1037 $\AA$ ), we have included in this analysis the modeling of the CIV resonance lines. These UV lines are sensitive to the location, size and temperature of the chromosphere above the photosphere, as demonstrated in the case of the prototypical Herbig Ae star AB Aur by Bouret \& Catala (1998). We have computed line profiles for all these ions that probe different regions and physical conditions in the chromospheric model. Finally, we have calculated the $\mathrm{Mg}$ II $\mathrm{h}$ and $\mathrm{k}$ resonance lines predicted for our chromospheric models to assess the influence of the chromosphere. Indeed, $\beta$ Pictoris reveals purely photospheric Mg II resonance lines (a weak chromospheric core might be washed out due to relatively fast rotation). The Mg II lines could thus constrain the chromospheric models, setting a limit on the depth of the region where the chromospheric temperature rise could start.

Hereafter, we briefly recall (and update when necessary) the conclusions concerning the apparent structure of the lines profiles that are relevant to our study, as they have been discussed in Vidal-Madjar et al. (1994) and Deleuil et al. (2001). Table 1 summarizes the observations.

Table 1. Summary of observational data.

\begin{tabular}{lcc}
\hline \hline Instrument & Date & $\begin{array}{c}\text { Exposure Time } \\
(\mathrm{s})\end{array}$ \\
\hline STIS-E230H..... & December 6 1997 & 360 \\
STIS-E140H..... & December 6 1997 & 900 \\
FUSE.............. & March 1/3, 2001 & $28000^{\mathrm{a}}$ \\
\hline
\end{tabular}

a Total exposure time, for the two days of observations.

\subsection{FUSE data}

The March 2001 dataset that we discuss here has been reprocessed using the version 2.0.4 of calfuse, the FUSE pipeline processing software. This version provides a better calibration and corrects for the sign error in the calfuse heliocentric velocity calculation. The stellar continuum, which is only detected down to $\sim 1100 \AA$, is well reproduced by a Kurucz model for $\beta$ Pictoris (see Lanz et al. 1995). Comparing the position of a few C I photospheric lines predicted by this Kurucz model to those observed on the Lif 2a spectrum near $1140 \AA$ A, we checked the absolute wavelength scale of our FUSE spectra. The resulting uncertainty in the wavelength calibration is less than $20 \mathrm{~km} \mathrm{~s}^{-1}$. Finally, we overplotted Sic 1a and Lif 1a channels and checked that they match within $5 \mathrm{~km} \mathrm{~s}^{-1}$, the comparison being achieved in the wings of O VI $\lambda 1032$ line, which has the highest signal to noise ratio in this wavelength range. This operation ensures that the absolute wavelength calibration in Sic and Lif channels is well established.

Table 2 summarizes some of the major properties of the emission lines (in order of increasing ionization), that we discuss hereafter. 


\subsubsection{Carbon lines}

The C III resonance line at $977 \AA$ appears as a broad, non Gaussian emission feature, with a total width of about $950 \mathrm{~km} \mathrm{~s}^{-1}$ (Fig. 1). Absorption components, whose origin and nature are not clear, are superimposed on the emission profile. A deep, broad $(0.2 \AA)$ depression affects the center of the profile at $977.15 \AA$. This component is seen on both Sic $1 \mathrm{~b}$ and Sic 2 a channels. The wavelength of this absorption component corresponds to a redshift with respect to the stellar velocity of $30 \mathrm{~km} \mathrm{~s}^{-1}$ (Fig. 1). Both the important velocity shift and intensity of this central component make it unlikely to be of interstellar origin. Indeed, few local ISM absorption features are seen toward $\beta$ Pictoris in Fe II or other lines in STIS spectra; they are weak and are measured at $-9 \mathrm{~km} \mathrm{~s}^{-1}$ with respect to the stellar velocity. A second absorption component is seen at $976.5 \AA$ on the Sic 2 a spectrum, but is less obvious on the Sic $1 \mathrm{~b}$ spectrum, suggesting that it may be an observational artefact. The wavelength of this absorption corresponds to a blueshift of $-200 \mathrm{~km} \mathrm{~s}^{-1}$ with respect to the stellar velocity (Fig. 1).

The CIII UV4 multiplet (a blend of six components at $\lambda 1174.933, \lambda 1175.263, \lambda 1175.59, \lambda 1175.711, \lambda 1175.987$, $\lambda 1176.37)$ is well detected in both Lif $1 \mathrm{~b}$ and Lif $2 \mathrm{a}$ spectra. Unlike the complex profile of the ion C III resonance line, the shape of this multiplet is Gaussian-like and the fit shows that the line is blueshifted by about $220 \mathrm{~km} \mathrm{~s}^{-1}$ relative to the stellar velocity (Fig. 1). This global blueshift is further confirmed by looking at the individual components of the multiplet, each of them being blueshifted by $220 \mathrm{~km} \mathrm{~s}^{-1}$ in the star's rest frame. The peak of the observed feature corresponds to the $\lambda 1175.711$ transition, the strongest of the multiplet.

A weak emission line at $1035.8 \AA$, blended with the blue wing of the O VI $\lambda 1037$, most likely corresponds to the $\mathrm{C}$ II resonance line at $1036.3 \AA$ blueshifted by $130 \mathrm{~km} \mathrm{~s}^{-1}$. On the other hand, the detection of both this resonance line and the excited line at $1037 \AA$ in absorption at their rest wavelength is much more uncertain, though they might correspond to the weak absorption troughs on the blue wing of O VI $\lambda 1037$. Observing them as two independent components suggests an interstellar or circumstellar, rather than stellar, origin for these lines. Indeed, if formed close to the star, they would be affected by the high rotation rate of $\beta$ Pictoris and they would merge and create an artificial broad absorption feature, which is not the case (see e.g. Deleuil et al. 2001, Fig. 3).

\subsubsection{The O VI resonance doublet}

The OVI doublet lines have symmetrical profiles and could be reproduced by Gaussian profiles. A Gaussian fit has been made for both components, using a standard minimization procedure.The wavelength separation of the two components was the only fixed parameter. The line ratio $\lambda 1037 / \lambda 1032$ measured from the fit is equal to the theoretical ratio, showing that the gas is optically thin in this wavelength range. Should the absorption trough seen at $1037.1 \AA$ be caused by C II interstellar absorption, the wavelength scale in the Lif 1a channel would be further confirmed to be good within $5 \mathrm{~km} \mathrm{~s}^{-1}$. Compared to the previous analysis (Deleuil et al. 2001), this calibration revealed a much smaller velocity shift of $10 \mathrm{~km} \mathrm{~s}^{-1}$ (instead of $46 \mathrm{~km} \mathrm{~s}^{-1}$ ) for the two lines, relative to the star.

\subsection{HST/STIS UV data}

For the present work, we have used HST/STIS high resolution echelle spectra showing the spectral range around the C IV $\lambda 1548-1550$ and Mg II $\lambda 2796-2803$ resonance doublets (E140H and E230H gratings, respectively). These spectra have been described in Roberge et al. (2000). Errors in the velocity determination are about $1 \mathrm{~km} \mathrm{~s}^{-1}$, given the high accuracy in the absolute wavelength calibration of STIS. The reader is referred to Roberge et al. (2000) for more details about data processing.

\subsubsection{The Mg II resonance doublet}

These lines have been extensively observed in the past, though at lower spectral resolution and signal to noise. They appear always in absorption. In addition to the photospheric absorption lines, centered at rest wavelengths, there are time-variable, redshifted (up to $300 \mathrm{~km} \mathrm{~s}^{-1}$ ) components that are produced by the evaporation of FEBs (Vidal-Madjar et al. 1994).

\subsubsection{The CIV resonance doublet}

The two components of the doublet appear in absorption and are redshifted by $4 \mathrm{~km} \mathrm{~s}^{-1}$, with additional redshifted $\left(\sim 300 \mathrm{~km} \mathrm{~s}^{-1}\right)$ components. The time variability of the latter has been revealed by IUE (Deleuil et al. 1993), despite a quite low stellar flux and $S / N$ ratio. These obervations had been further confirmed with HST/GHRS observations (Vidal-Madjar et al. 1994). The two redshifted components have similar strengths, indicating that they are saturated.

Table 2. Emission line parameters as measured from our FUSE spectra. $f_{\lambda}$ is the emission line surface flux. Following Simon \& Ayres (1998), we also list the normalized flux $R_{\lambda}=f_{\lambda} / f_{\text {bol }}$ and the ratio of the latter to the corresponding solar values: Ratio $=$ $R_{\lambda}(\beta$ Pictoris $) / R_{\lambda}$ (Sun). Note that the lines with the lowest ratios are also those with the highest blueshifts, further suggesting that they are formed in a different medium than the other lines.

\begin{tabular}{cccccc}
\hline \hline Line & $\begin{array}{c}\lambda \\
(\AA)\end{array}$ & $\begin{array}{c}V^{\mathrm{a}} \\
\left(\mathrm{km} \mathrm{s}^{-1}\right)\end{array}$ & $\begin{array}{c}f_{\lambda} \\
\left(\mathrm{ergs} \mathrm{cm}^{-2} \mathrm{~s}^{-1}\right)\end{array}$ & $\begin{array}{c}R_{\lambda} \\
\left(10^{-7}\right)\end{array}$ & Ratio \\
\hline C II $^{\mathrm{b}}$ & 1036 & -130 & $1290 \pm 30$ & 0.05 & 0.18 \\
$\mathrm{C} \mathrm{III}^{\mathrm{c}}$ & 977 & $\ldots$ & $50000 \pm 400$ & 2.03 & 1.40 \\
$\mathrm{C} \mathrm{III}^{*}$ & 1176 & -220 & $5800 \pm 130$ & 0.23 & 0.37 \\
O VI & 1032 & 10 & $14500 \pm 193$ & 0.59 & 0.81 \\
O VI & 1037 & 10 & $6900 \pm 241$ & 0.28 & 0.45 \\
\hline
\end{tabular}

a Velocity shift of the central emission peak, with respect to the star's radial velocity.

${ }^{\mathrm{b}} R_{\lambda}(\mathrm{Sun}) \approx 2.8 \times 10^{-8}$ (Th. Ayres, private communication).

${ }^{\mathrm{c}}$ Cannot be modeled as a single Gaussian profile. 


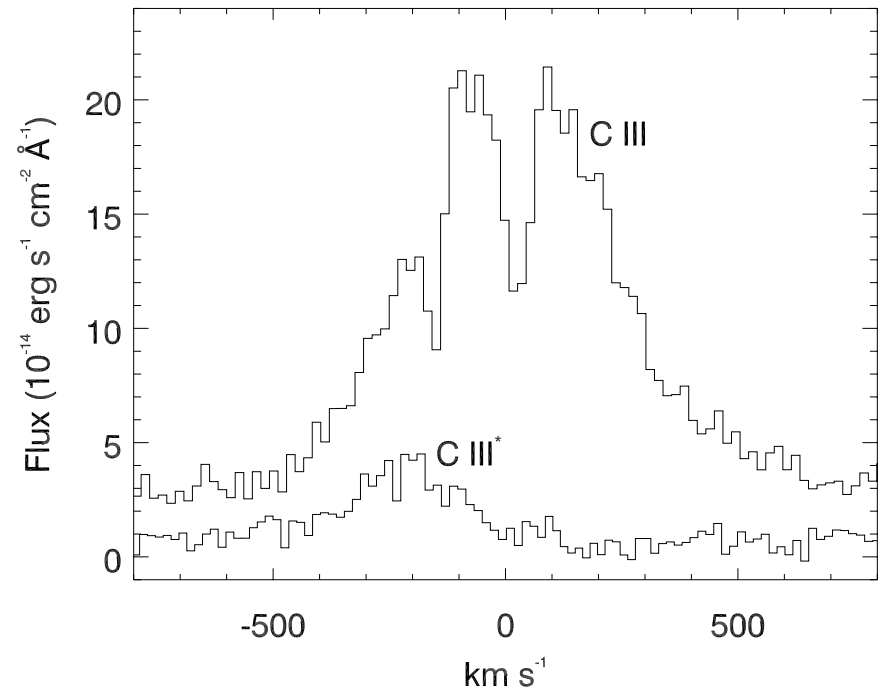

Fig. 1. FUSE spectrum of $\beta$ Pictoris showing the CIII (Sic 2a) and $\mathrm{CIII}^{*}$ lines (Lif 2a) in emission. The $x$-axis shows velocities (in $\mathrm{km} \mathrm{s}^{-1}$ ) with respect to the stellar heliocentric velocity. The $y$-axis is in units of $10^{-14} \mathrm{ergs} \mathrm{cm}^{-2} \mathrm{~s}^{-1} \AA^{-1}$ for both ions, but C III has been shifted by +3 for the sake of clarity.

\section{Basic ingredients for the modeling}

\subsection{Fundamental parameters and photospheric models}

Using HST/GHRS observations combined with stellar atmosphere modeling and spectral synthesis techniques, Lanz et al. (1995) derived reliable parameters for $\beta$ Pictoris: $T_{\mathrm{eff}}=8200 \pm$ $150 \mathrm{~K}, \log g=4.25 \pm 0.1, L_{\mathrm{bol}}=11.3 \pm 3.5 L_{\odot}$ and $M=$ $1.8 \pm 0.1 M_{\odot}$. From these values, a straightforward estimate of the stellar radius is $R=1.67 \pm 0.1 R_{\odot}$. These authors also showed that elemental abundances are solar-like and that the rotation velocity of the star is $v \sin i=140 \mathrm{~km} \mathrm{~s}^{-1}$, which we adopted throughout this work.

We further assume that $\beta$ Pictoris photosphere is adequately described by a classical Kurucz model atmosphere, in radiative and hydrostatic equilibria, with the above-mentioned parameters.

We adopted $v_{\text {turb }}=2 \mathrm{~km} \mathrm{~s}^{-1}$ as a conservative value for the microturbulent velocity of the model. Other values have been tested, always lower than the sound speed in the photosphere $\left(c_{\mathrm{s}} \approx 10 \mathrm{~km} \mathrm{~s}^{-1}\right.$ for $\beta$ Pictoris) but, because of the high rotational broadening, this parameter has no visible effect on the synthetic profiles.

We will always adopt the same description of the photospheric layers, making a comparison between our different models (photosphere+chromosphere+transition region) dependent only on the physical conditions in the outer atmosphere.

\subsection{Chromospheric models}

Our modeling of the hot region surrounding $\beta$ Pictoris is directly inspired from the semi-empirical approach developed by Bouret \& Catala $(1998,2000)$ to study the activity of Herbig $\mathrm{Ae} / \mathrm{Be}$ stars. On the top of the photosphere, a model for the extended heated region where C III, C IV and O VI are expected to form is appended. Adopting a solar analogy, we will refer to the different parts of this zone as chromosphere and transition region (hereafter TR), to distinguish the formation region of C III and C IV resonance lines on the one hand and the one of $\mathrm{O}$ VI resonance lines on the other hand. We assume this region to be spherically symmetric when calculating the various quantities describing the model.

\subsubsection{Temperature structure}

The temperature law from the Kurucz model is adopted in the photosphere until it reaches a specified minimum $T_{\min } \leq T_{\text {eff }}$. Since, obviously, this parameter has no influence on the line profiles of the highly ionized species we are interested in here, we fixed it once for all, after studying its effect on photospheric lines.

In the chromosphere and transition region, we adopted temperature laws sharply rising outside the photosphere and extending radially for a few tenths of stellar radii. Using different lines of different ions with different formation conditions allowed us to tightly constrain the temperature law required to reproduce the shapes and intensities of the observed line profiles. More precisely, temperature regimes in the chromosphere have been constrained through the analysis of C II, C III and $\mathrm{C}$ IV resonance lines. They probe temperatures roughly ranging from 17000 to $100000 \mathrm{~K}$. We first built a model with a constant temperature gradient, from the bottom $\left(T=T_{\min }\right)$ to the top $(T=130000 \mathrm{~K})$ of the chromosphere. This maximum temperature was determined as the upper limit above which the ionization equilibrium shifts from $\mathrm{CIV}$ to $\mathrm{CV}$. The location of the outer chromospheric point was chosen such that, if the gas is on Keplerian orbits around the star, the linear velocity on the corresponding radius is three times $v \sin i$ as suggested from the width of C III and O VI lines. This model failed to reproduce C II, C III and C IV lines as observed. Models with variable temperature gradients were then explored.

Furthermore, the mere presence of O VI lines in a stellar spectrum indicates a temperature higher than $160000 \mathrm{~K}$ in the line formation region. The temperature law in the transition region has been built as follow: at the bottom of the TR, the first point of the temperature is the upper point of the chromospheric model giving the best agreement with the carbon ions lines. The outside most point of the TR was fixed such that after this point, whatever temperature law is adopted, no visible contribution is made to the O VI lines already modeled. The simplest TR model, including a single, constant gradient throughout the whole region had to be rejected because no satisfactory agreement could be found with the observed O VI doublet profiles. As for the chromosphere case, models with two gradients in the TR were explored.

In summary, the principal free parameters describing the line formation regions are their size and location and the temperature gradients between the photosphere and the external point of the TR. In the remainder of the paper, we will use the column mass $m=\rho \mathrm{d} r$ to conveniently locate the different zones of the temperature law. Locations of the different 
temperature gradients have been fixed successively, starting from the base of the chromosphere and proceeding outwards, so as to fit our sample of lines. Varying the steepness of the gradient and the size of the region over which this gradient is applied makes it possible to study the effect of an accumulation of gas at formation temperatures of a given line.

\subsubsection{Density structure}

The density distribution $\rho(r)$ in the chromosphere-transition region complex is obtained by extrapolating the hydrostatic equilibrium structure of the underlying photosphere Kurucz model. We then compute the velocity law through the continuity equation $v(r)=\dot{M} / 4 \pi r^{2} \rho(r)$, where $\dot{M}$ is the a free parameter describing the outflow rate in the model. With density decreasing monotonically outwards, this equation shows that the velocity law is an increasing function of the radial distance $r$ from the stellar center. A smooth connection bewteen the velocity law in the chromosphere-TR and the photosphere is obtained by requiring that at the connecting point, we have:

$\frac{\tau_{\star}}{\int_{R_{\mathrm{ph}}}^{\infty} \frac{\sigma_{\mathrm{c}}(r)}{r^{2} v(r)} \mathrm{d} r}=R_{\mathrm{ph}}^{2} \rho_{\mathrm{ph}} v\left(R_{\mathrm{ph}}\right)$

$\tau_{\star}$ being the continuum optical depth at the photospheric radius $R_{\text {ph }}$ while $\rho_{\text {ph }}$ is the density of the photospheric model at the adopted $\tau_{\star} . \sigma_{\mathrm{c}}$ is the continuum absorption cross-section per mass unit (in $\mathrm{cm}^{2} \mathrm{~g}^{-1}$ ), including electron scattering. Since observations show that photospheric lines are well reproduced by the Kurucz model we have adopted here, we assume $\tau_{\star}=2 / 3$ at $r=R_{\mathrm{ph}}$.

For all the models, we adopted a canonical $\dot{M}$ that we obtained as follow. Assuming that the gas in the chromospheric region is fully ionized, we wrote that $\rho=1.4 m_{\mathrm{H}} n_{\mathrm{e}}$ where $m_{\mathrm{H}}$ and $n_{\mathrm{e}}$ are the mass of an hydrogen atom and the electron density, respectively. Then, $n_{\mathrm{e}}$ was determined from the flux ratio $f_{1176} / f_{977}$ which is well known to be sensitive to electron density over the range $7 \leq \log n_{\mathrm{e}} \leq 11$ (Bathia \& Kastner 1993). No C III* line, close to its rest wavelength, is seen on the FUSE data; therefore, we could only estimate an upper limit on the flux ratio $f_{1176} / f_{977} \leq 0.1$. Using theoretical curves from Bathia \& Kastner (1993) for a standard formation temperature for ion C III $(\log T=4.8$, Simon \& Ayres 1998), we concluded that $\log n_{\mathrm{e}} \leq 7.5$. For a velocity of a few tens of kilometer per second, corresponding to the thermal speed for the adopted temperature, we obtained: $\dot{M} \approx 2.5 \times 10^{-14} M_{\odot} \mathrm{yr}^{-1}$. This value is consistent with the theoretical work of Babel (1995) on the mass loss rates of mainsequence A-type stars and with the observations of Bruhweiler et al. (1991) on $\beta$ Pictoris. It is also compatible with what was found by Beust et al. (2001), when studying the interaction between an outflowing wind and infalling evaporating cometary bodies. They showed that the upper limit above which no spectral signatures for such bodies could be detected in the $\beta$ Pictoris system is $\dot{M} \approx 1 \times 10^{-11} M_{\odot} \mathrm{yr}^{-1}$. Note that such an upper limit on the mass loss rate was found by Lanz \& Catala (1992) when searching for traces of stellar winds in $\mathrm{H} \alpha$ profiles of a sample of main-sequence AV stars. Furthermore, as far as the line profiles are concerned, we found no significant differences for models with $1 \times 10^{-14} \leq \dot{M} \leq 1 \times 10^{-12} M_{\odot} \mathrm{yr}^{-1}$.

\subsection{Radiative transfer and atomic models}

Line profiles are calculated with the ETLA code, which solves the radiative transfer equation in the comoving frame of the gas, in the equivalent two-level-atom formulation introduced by Mihalas \& Kunasz (1978). NLTE effects for lines and continua of interest are taken into account (Bouret \& Catala 2000). In a first step, ETLA solves the transfer equation together with the equations of statistical equilibrium for three successive ionization stages, in the comoving frame formulation. Once this step has been performed, calculations in the observer's frame give the emergent flux in the transitions we are interested in, as well as many other physical quantities, such as the continuum flux.

The atomic model that we have used for carbon is made of 7 levels for C II, 8 levels for C III and C IV and one continuum level for C V. The HI and Mg II models are similar to those used in Bouret \& Catala (1998). For the computation of the O VI lines, we have considered 6 levels for O V, 13 levels for $\mathrm{OVI}$ and again, one continuum level for O VII.

For all these ions but H I, Doppler profiles have been assumed (in the atomic rest frame). We have introduced the calculation of the Stark's wings for the $\mathrm{H} \alpha$ line, in the observer's frame step.

Auto-ionizations have been neglected but dielectronic recombinations are taken into account. We have assumed complete redistribution for all lines. This latter assumption may not be justified for the case of $\mathrm{Mg}$ II $\mathrm{h}$ and $\mathrm{k}$ lines, since partial redistribution effects have been shown to be potentially significant for some species in static atmospheres (see Mihalas 1978 or Hubeny 1985 for the case of MgII h and $\mathrm{k}$ lines). Here however, these effects are likely to be smaller than the effect of Doppler-shift induced by the gas macroscopic motion.

Several chemical species, in various ionization stages, are considered to compute the background continuum. Namely, we used H (bound-free), He I, He II, C I to C IV, N II, N III, N IV, O II, O III, O IV, Ne II, Mg I, Al I, Si I to Si IV. Their contributions are computed assuming LTE.

\subsection{Computed line profiles and observations}

To compare our predicted line profiles to observations, we need still to properly normalize the theoretical spectra and account for the stellar rotation and the star's radial velocity.

Because of the assumption of spherical symmetry when solving the radiative transfer in the comoving frame, rotation of the envelope can be introduced only in the observer's frame step. Convolution with an analytical rotational profile is performed in order to get line profiles as broad as observed. This operation is acceptable, given that the chromospheric line formation region is very narrow.

The observed profile is corrected for the reddening caused by the interstellar medium, using $E(B-V)=0.02$ and the 
reddening curve from Cardelli et al. (1989); this yields the real flux in the emission lines.

The synthetic flux, calculated at the outer limit of the chromospheric region, is converted to flux at earth, adopting a distance of $19.3 \mathrm{pc}$.

Finally, all the spectra (i.e. FUSE and HST/STIS) have been shifted by $-20 \mathrm{~km} \mathrm{~s}^{-1}$ to take into account the radial velocity of the star.

\section{Line profiles in the chromospheric scenario}

We focussed on models producing single peak emission profiles for C III. Double-peaked emission generally forms in rotating circumstellar envelopes or disks. In this case, the interpeaks separation is $\Delta v=2 v \sin i$. For $\beta$ Pictoris one would therefore expect $\Delta v \approx 280 \mathrm{~km} \mathrm{~s}^{-1}$. Measuring the actual separation between the two most intense peaks with our new wavelength calibration, we find $\Delta v=205 \mathrm{~km} \mathrm{~s}^{-1}$, which therefore seems to rule out this interpretation. Even though doublepeaked emission profiles can form in chromospheric models, producing them with their central absorption shifted with respect to the rest wavelength requires significant mass loss rates as well as high velocities projected on the line of sight (Bouret \& Catala 2000), in contradiction with our present assumptions. This lead us to assume that absorbers located outside the line formation region are responsible for the two absorption components seen on the line profile. The nature of these absorbers will be discussed later on.

\subsection{Mg II lines profiles}

The minimum of temperature, $T_{\min }$, was obtained by modeling the Mg II resonance lines. Line wings of the latter are formed quite deep in the photosphere while for wavelengths around the line core, the flux is coming from the upper photosphere, near the temperature minimum. Exploring the dependance of the synthetic profiles with $T_{\min }$, we obtained a good agreement between synthetic profiles and observations for $T_{\min }=5350 \mathrm{~K}$. We also systematically checked that whatever parameters we adopted for the chromosphere + TR model, the Mg II line profiles remained mostly unchanged and are similar to those resulting from the Kurucz model (see Fig. 2).

These profiles agree reasonably well with the HST/STIS observations, given that the observed profiles are highly contaminated by non stellar contributions. In particular, producing realistic spectra of these lines implies that absorption by the gas of the massive circumstellar disk of $\beta$ Pictoris is taken into account. The disk indeed produces well-known absorption features in the cores of low-excitation metal lines (Kondo \& Bruhweiler 1985; Hobbs et al. 1988; Vidal-Madjar et al. 1994). As shown by Lanz et al. (1995), once introduced in the spectrum modeling, the circumstellar gas absorption allows one to achieve a remarkably good fit of most of the profiles, with the exception of the redshifted absorption attributed to FEBs contributions.

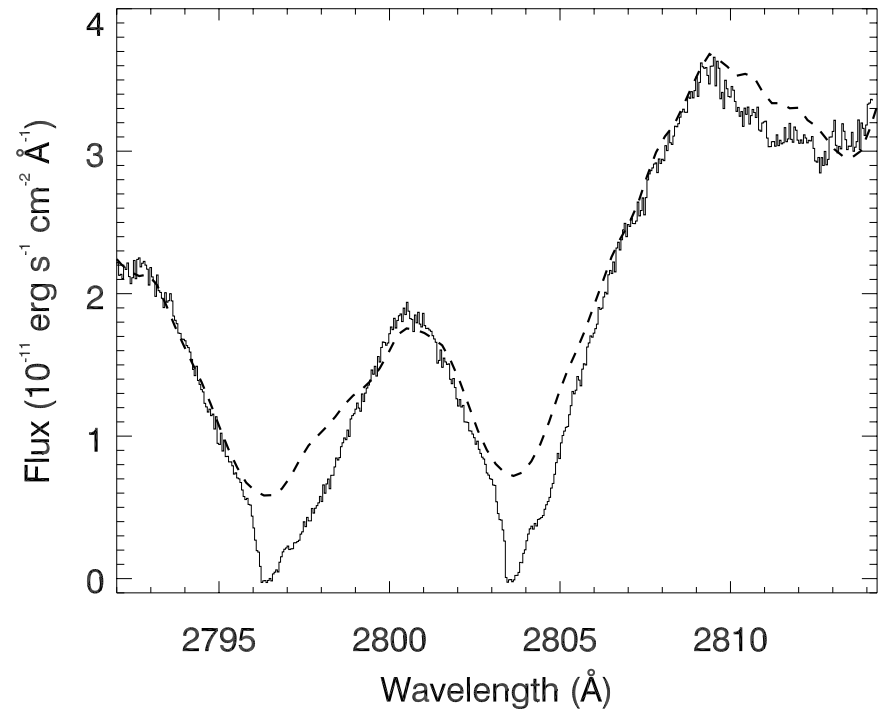

Fig. 2. Mg II: Comparison between HST/STIS profiles (full line) and profiles resulting from Kurucz photospheric model (dashed line).

\subsection{Carbon and O VI line profiles}

The models we discuss hereafter have the same photospheric stucture, obtained from the modeling of Mg II. The chromospheric temperature laws start at the minimum of temperature $\left(\log m=-3.44, T_{\min }=5350 \mathrm{~K}\right)$.

C II lines form in absorption for models in which the temperature is higher than $T=13000 \mathrm{~K}$ and lower than $T=$ $19000 \mathrm{~K}$ for $\log m=-3.56$ and $\log m=-3.73$ respectively. Below $T \sim 13000 \mathrm{~K}$, there is almost no C II left; therefore, with the present data set, we lack information to constrain the temperature in the deepest, coolest, region of the chromosphere, connecting the photosphere to the C II formation region. Because of the higher gas density prevailing in the deep chromosphere, where these lines form, they are optically thick. As a consequence, we cannot compare our synthetic profiles to the observed ones by simply adding them to the the blue wing of the $1036 \AA$ O VI line (see Sect. 2.1.1). ETLA is not suitable to treat line blends which prevent us from taking into account the effect of the underlying line and to compare the resulting C II line profiles to observations.

C III resonance line forms in emission in models with temperatures reaching up to $70000 \mathrm{~K}$ at the external border of the heated region. This maximum temperature corresponds to the threshold above which C IV becomes the dominant state of the ionization equilibrium. The size of the emitting region acts on the line intensity through a density effect: the farther (from the photosphere) the external border, the lower the gas density and therefore the number of emitting atoms. Numerically, we found this external border to be at $\log m=$ -4.3 .

As a consequence of the low electron density in the chromosphere resulting from the adopted value of $\dot{M}$, the C III* line is always quite weak, with a peak of intensity at less than 1.5 times the local continuum. Computing a detailed model of the underlying photosphere, we found that in the spectral range bracketting the C III* multiplet, the photospheric spectrum is 
dominated by absorption lines of Fe I. The closest to the C III* wavelength is also the strongest. Once the photospheric spectrum is convolved with $v \sin i=140 \mathrm{~km} \mathrm{~s}^{-1}$, this line is still quite strong and it can significantly affect the relative apparent intensity of the C III* line. We could not calculate the synthetic profile resulting from this blend with ETLA and compare it to the corresponding observed spectral region, but we argue that this can explain why no emission line is seen at C III* rest wavelength on our FUSE data.

Finally, once having fixed the limits for C II and C III lines formation region, we searched for the conditions to get $\mathrm{C}$ IV in absorption. Models with maximum temperature around $105000 \mathrm{~K}$ and with radial extension of $\Delta \log m=-0.8$ (starting at $\log m=-4.3$ for temperatures around $70000 \mathrm{~K}$ ) produce a high enough optical depth in the CIV formation region for the line to form in absorption. The theoretical C IV lines only fit the bluest part of each component of the observed doublet, known to be stable in time (see Fig. 5). The reddest parts are not reproduced at all, confirming the current interpretation that they are caused by FEBs.

As already stated, O VI lines indicate temperatures typically ranging from about $200000 \mathrm{~K}$ to $350000 \mathrm{~K}$. We have no observational data to constrain the temperature between the region where C III and C IV lines form and the O VI doublet formation zone. An accurate modeling of this region will have to wait for HST/STIS observations of the $\mathrm{N} \mathrm{V}$ resonance doublet (see Sect. 5.2).

We found that the models in which a steep rise of the temperature is followed by a much flatter gradient extending up to the top point of the TR give the best agreement with our observations.

For all these models, we checked that O VI lines are unchanged by modifications of the parameters of the inner chromosphere, as long as the location of the bottom of the TR and its temperature to a lesser extent is kept unchanged. Besides, stronger lines are obtained as temperatures higher than $200000 \mathrm{~K}$ are reached in regions closer to the star's surface, where the density is higher. This allowed us to proceed by trial and error to search for a global model fitting the O VI doublet together with the C III and C IV lines. Each line profile has been convolved with a rotational profile with an effective rotational velocity adapted for each element: $v_{\mathrm{eff}}=290 \mathrm{~km} \mathrm{~s}^{-1}$ for C III, $v_{\text {eff }}=310 \mathrm{~km} \mathrm{~s}^{-1}$ for C IV and $v_{\text {eff }}=400 \mathrm{~km} \mathrm{~s}^{-1}$ for O VI. Such a super-rotational broadening is compatible with the assumption that the gas is on a Keplerian orbit around the star at the distance where the lines form. We obtained a good agreement with the observed profiles for a model whose characteritic paramaters are listed in Table 3; Fig. 3 illustrates the temperature law of this model and shows the different regions of formation for the lines of interest. The corresponding profiles are shown in Figs. 2, 4, 5 and 6.

\subsection{Intrinsic versus apparent shape of the C III line}

The global shape of the profile of C III resonance line is well reproduced although some discrepancies remain, mainly due to the deep absorption features seen on the profile. As already said
Table 3. Parameters for the model producing the line profiles of Figs. 4, 5 and 6 (see text).

\begin{tabular}{ccccccc}
\hline \hline $\log m$ & -6.50 & -5.23 & -5.05 & -4.17 & -3.66 & -3.44 \\
$T$ & 400000 & 260000 & 100000 & 68000 & 26000 & 5350 \\
\hline
\end{tabular}

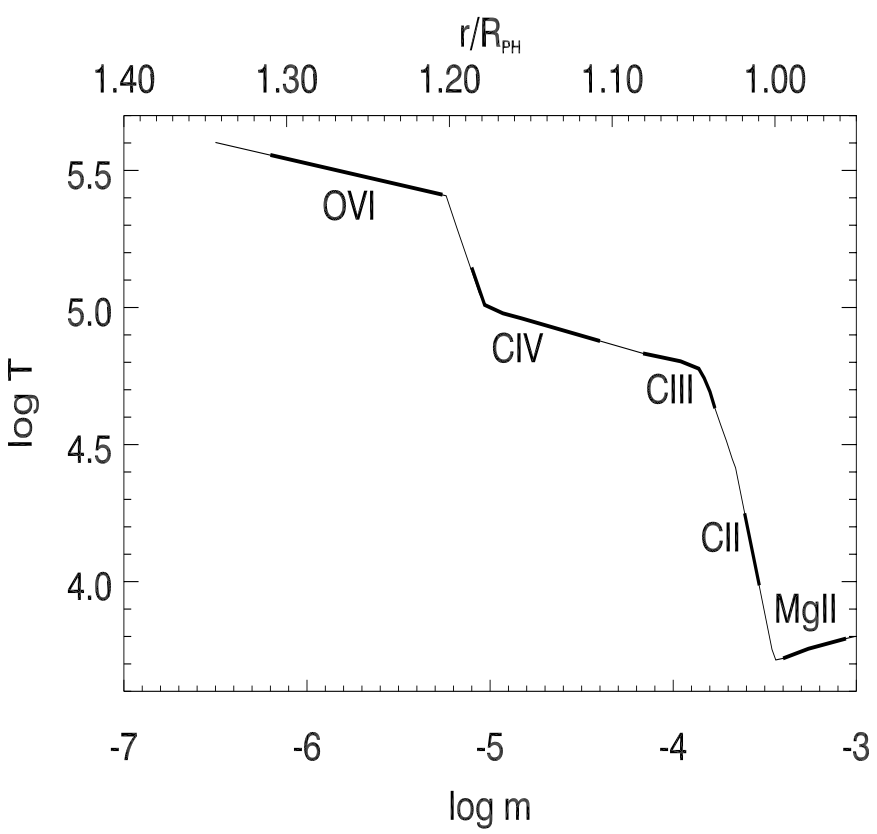

Fig. 3. Temperature (K) for the model of Table 3, as a function of the column mass $\left(\mathrm{g} \mathrm{cm}^{-2}\right)$; the upper $x$-axis shows the radial distance above the star, in photospheric radius units. The location of the different line formation regions are indicated.

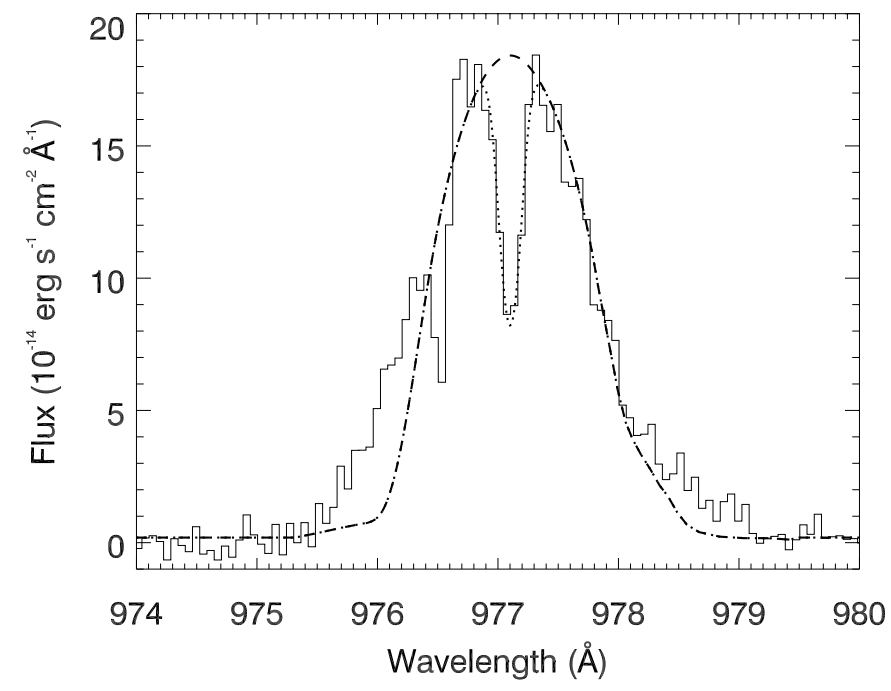

Fig. 4. Observed profile of C III $977 \AA$ (full line) compared to the profile obtained with the model from Table 3 (dashed line). The dotted line shows this synthetic profile + a superimposed absorption caused by a density column $\log n_{\mathrm{CIII}}=13.11$.

in Sect. 2.1.1, the bluest one may be an artefact. Nevertheless, we checked that it cannot be caused by $\mathrm{H} 2$ transitions at $976.55 \AA$, as it would imply an H 2 column density more than one order of magnitude higher than what was recently measured by Lecavelier des Etangs et al. (2001). Considering the 


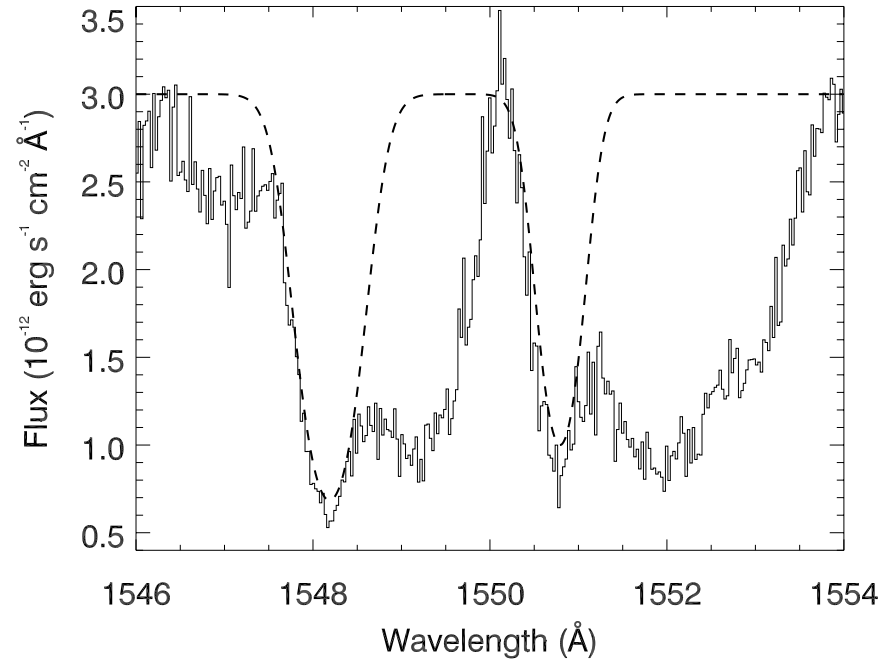

Fig. 5. Profiles obtained with the model from Table 3 for C IV doublet (dashed line) compared to the observed profiles (full line). Deep components centered on the rest wavelengths are produced by the chromospheric model.

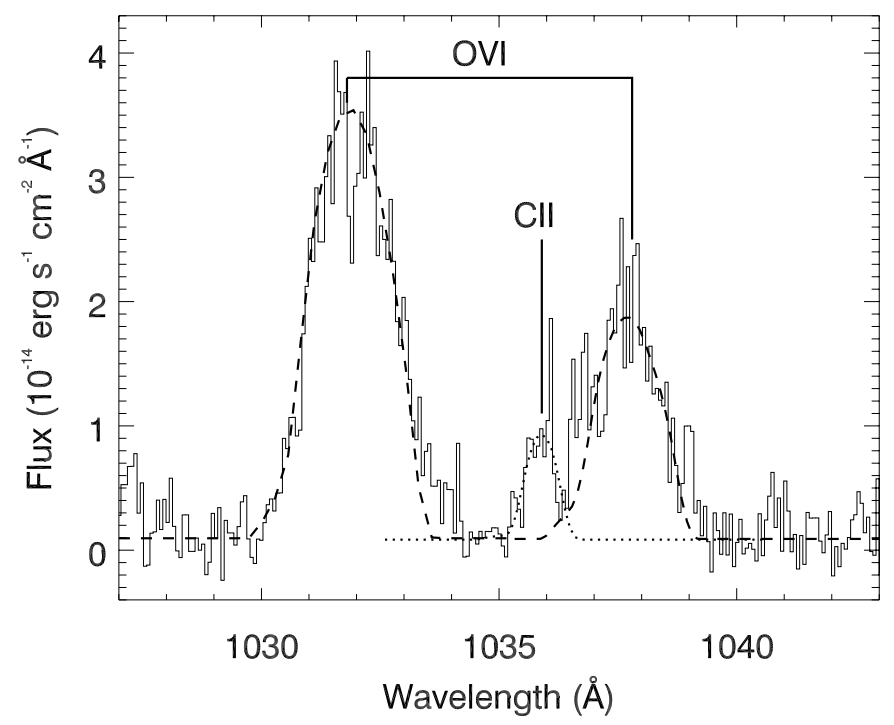

Fig. 6. The synthetic OVI doublet (dashed line), as obtained from model listed in Table 3, compared to the observed profiles (full line) in the Lif 1a spectrum. The dotted line is for the C II resonance line as produced by the disk-wind model discussed in Sect. 5. This fit suggests that the absorption feature at $1037.1 \AA$ is caused by CS C II (fine structure line) absorption.

low level of confidence for the real presence of this absorption at $-200 \mathrm{~km} \mathrm{~s}^{-1}$ on C III, we did not investigate in more detail any other possible origin. On the other hand, we have considered several possibilities for the nature and origin of the central absorption feature, unchanged in Sic $2 \mathrm{a}$ and Sic $1 \mathrm{~b}$ spectra.

Unresolved $\mathrm{CO}$ bands could be ruled out for two reasons: first, we found that a $\mathrm{CO}$ column density 100 times higher than that determined by Roberge et al. (2000) from HST/STIS data is needed to get an absorption component with the right strength for the Q branch from the $977.418 \AA$ band. Second, this band would have to be blueshifted by about $100 \mathrm{~km} \mathrm{~s}^{-1}$ to fall at the wavelength of the observed central absorption, which we considered unlikely given the conclusions of Roberge et al. (2000).

As shown by Beust \& Tagger (1993), highly ionized species such as Al III or C IV can form in the comae of FEBs; therefore, some C III must be produced as well. Although very appealing, this interpretation may be in contradiction with the fact that the observed absorption does not vary significantly on a short time scale. The observed trough could as well result from the presence of circumstellar (within the CS disk) and interstellar C III on the line of sight. Assuming that the central depression is indeed caused by the presence of C III on the line of sight, whatever its real origin, we can derive how much gas is involved. Using simple (no dynamics or kinematics) assumptions, we were able to reproduce fairly well the observed profile for a density column $\log n_{\mathrm{CIII}}=13.11$ and $v=30 \mathrm{~km} \mathrm{~s}^{-1}$ for the radial velocity of the absorbing gas (Fig. 4). Remaining differencies, affecting the blue wing of the profile, will be discussed in Sect. 5.

\section{Origin of the blueshifted emission lines}

\subsection{Observations and interpretation}

As already stated in Sect. 2.1, both the C II resonance line and C III excited line appear in emission and are blueshifted by 130 and $200 \mathrm{~km} \mathrm{~s}^{-1}$ respectively. Therefore, they cannot be formed in the chromospheric stucture we have assumed so far; the gas velocity in the chromosphere and in the TR is far from being high enough to induce such a high blueshift.

Although Beust \& Morbidelli (2000) have shown that models involving FEBs can produce blueshifted features, a FEBs origin could easily be ruled out for these lines. First, the line profiles are symmetric (Gaussian) while the FEBs model predicts that lines with high velocity shifts are strongly asymmetric. Second, the lines do not vary on a short time scale (hours), nor do they on a long time scale (a few days).

Whatever the mechanism producing the observed blueshift, the FUSE observations tell us that velocities as high as $200 \mathrm{~km} \mathrm{~s}^{-1}$ are reached in the line formation region and that the flow is stable over time. A simple interpretation of the observed velocity shift is to assume that the lines form in a flow, blown from the $\beta$ Pictoris gazeous disk. This disk wind has to be warm enough to produce C III*. On the other hand, the maximum temperature in the wind must be low enough that neither C IV nor O VI components are formed. If the disk wind hypothesis is correct, the FUSE observations show that the vertical size of the line formation region (measured perpendicularly to the disk plane) must be such that it is smaller than the stellar disk. Indeed, the observed profiles are fully blueshifted, with no extension at redshifted wavelengths, which shows that if formed in an expanding wind, the receeding part of the latter does not contribute to the line profile. This has two straightforward consequences: first, the temperature for the production of C III must be reached not far from the disk plane. Second, the density of the wind must decrease drastically outside the line formation region, such that no blueshifted absorption lines from other species, like Fe I, Na I or Balmer lines, are observed, contrary to the well documented case of FU Ori disk winds 
(e.g. Calvet et al. 1993; Hartmann \& Calvet 1995). Finally, despite the C II and C III* lines likely forming close to the CS disk, they show no evidence of rotation, which indicates that the expansion velocity field dominates over the rotation velocity of the disk, in the line formation region.

This interpretation is further strengthened by turning back to the C III resonance line profile. The blue side of the theoretical line is not well reproduced by the chromospheric model and some additional emission is seemingly missing. More precisely, there is a peak at $976.37 \AA$ which exactly corresponds to a blueshift of $200 \mathrm{~km} \mathrm{~s}^{-1}$ for the $977.02 \AA \mathrm{C}$ III line. It is very appealing to interpret this additional emission for the C III resonance as being produced in the same region as the $\mathrm{C}$ III excited lines.

\subsection{A disk-wind model}

To test whether this scenario could explain the observed characteristics of the carbon blueshifted lines, we built a model in which a region, much hotter than the surrounding CS gas, is arbitrarily located at ten stellar radii from the star and extends over a few stellar radii along the disk plane.

The adopted temperature law is composed of three distinct parts: first, a steep rise above the disk plane, then a temperature plateau at $T_{\max }$ and finally a regime where the temperature steeply decreases outwards until it reaches a terminal value $T_{0}$. The wind therefore ends up with an isothermal cool region. $T_{0}=20 \mathrm{~K}$ was adopted, consistent with what is known of the $\beta$ Pictoris CS gaseous environment (Vidal-Madjar et al. 1998). Four free parameters $\left(H_{\mathrm{p}}, T_{\max }, \Delta_{1}, \Delta_{2}\right)$ govern this temperature law, which is expressed as follow:

$$
T(z)=\left\{\begin{array}{l}
T_{0}+\left(T_{\max }-T_{0}\right) \exp \left[-4 \ln 2 \frac{\left(z-Z_{\mathrm{p} 1}\right)^{2}}{\Delta_{1}^{2}}\right] \\
\text { if } Z_{\mathrm{D}} \leq z \leq Z_{\mathrm{p} 1} \\
T_{\max } \\
\text { if } Z_{\mathrm{p} 1} \leq z \leq Z_{\mathrm{p} 2} \\
T_{0}+\left(T_{\max }-T_{0}\right) \exp \left[-4 \ln 2 \frac{\left(z-Z_{\mathrm{p} 2}\right)^{2}}{\Delta_{2}^{2}}\right] \\
\text { if } Z_{\mathrm{p} 2} \leq z .
\end{array}\right.
$$

The temperature plateau, where the maximum temperature is reached, starts at the point $Z_{\mathrm{p} 1}$ defined by:

$Z_{\mathrm{p} 1}=Z_{\mathrm{D}}+\frac{\Delta_{1}}{2(\ln 2)^{1 / 2}}\left[\ln \left(\frac{T_{\max }-T_{0}}{T_{\mathrm{D}}-T_{0}}\right)\right]^{1 / 2}$

and ends at the point $Z_{\mathrm{p} 2}=Z_{\mathrm{p} 1}+H_{\mathrm{p}} \cdot Z_{\mathrm{D}}$ and $T_{\mathrm{D}}$ are the CS disk thickness and its temperature, respectively, while $\Delta_{1}$ and $\Delta_{2}$ control respectively the position of the maximum temperature and the extension of the decreasing part of the temperature law. Following Lanz et al. (1995), we used $T_{\mathrm{D}}=5000 \mathrm{~K}$, while the disk thickness was assumed to be 0.2 stellar radii (cf. Goldreich \& Ward 1973)

Afterwards, we adopted a piecewise linear velocity law such that velocities ranging from $150 \mathrm{~km} \mathrm{~s}^{-1}$ to $250 \mathrm{~km} \mathrm{~s}^{-1}$ are reached in this region. Then, we integrated the line specific intensity only on rays intercepting the stellar disk. Variation of the line intensity are obtained by varying the temperature and the volume of the emitting region, i.e. extending it on the radial direction.

The electron density in the C III lines formation region was fixed by measuring the flux ratio $f_{1176} / f_{977}$ for the blueshifted observed lines. The contribution to the line flux $f_{977}$ from the disk-wind region was obtained by substracting the theoretical flux from the chromospheric model to the observed flux. We found $f_{1176} / f_{977}=0.51$ which corresponds to $n_{\mathrm{e}}=10^{10.2}$ for a gas temperature $T_{\max }=40000 \mathrm{~K}$, that we used at this step. Because of possible opacity effects in the lines, the estimated density is an upper limit to the true density of the medium. Assuming that the wind is fully ionized in the line formation region, we can write:

$\dot{M} \approx \frac{v_{\mathrm{w}}}{R_{\mathrm{w}}} \int 1.4 n_{\mathrm{e}} m_{\mathrm{H}} \mathrm{d} V \quad\left(M_{\odot} \mathrm{yr}^{-1}\right)$.

Here, $v_{\mathrm{w}}$ and $R_{\mathrm{w}}$ are the wind velocity (as measured from the observed blueshift) and the radial extent of the line formation region. Adopting the mass loss rate corresponding to each model, we derive the density distribution through the continuity equation and then calculate line profiles. When integrating the line profiles over the rays intercepting the stellar disk, we excluded those between the disk plane and the 0.2 stellar radii limit. The emitting region was started immediately above the disk external boundary. Varying both the radial and vertical extension, we searched for the values allowing to reproduce the observed profile. We found $1 \leq R_{\mathrm{W}} \leq 5 R_{\star}$ and $0.37 \leq h_{\mathrm{w}} \leq$ $0.8 R_{\star}$, where $h_{\mathrm{w}}$ is the vertical size of the wind emission region. Figure 7 shows the profile of C III* produced by a model with $R_{\mathrm{w}}=3.5 R_{\star}$ and $h_{\mathrm{w}}=0.65 R_{\star}$. For these parameters, we deduce a mass loss rate of $\dot{M}=6.6 \times 10^{-11} M_{\odot} \mathrm{yr}^{-1}$. The line profile for the $\mathrm{C}$ II resonance line produced by the same model is also plotted in Fig. 6. It further illustrate the ability of the disk-wind framework to explain the presence of blueshifted emission lines in $\beta$ Pictoris spectrum. Finally, we thoroughly checked that the C III resonance line appears in emission and is blueshifted in this model and produces a contribution that superimposes on the blue wing of the chromospheric C III line, as observed by FUSE.

Results hereabove strongly suggest that a disk-wind is indeed responsible for the observed blueshifted C II and C III* lines. Although based on simple assumptions, our modeling implies that the opening angle of the outflow, defined as the angle between the disk midplane and the maximum latitudinal extent of the outflow, is small. Such a result is very similar to what was found for the Herbig A0e star HD 100564 by Viera et al. (1999). However, in our case, an origin from the CS disk rather than from the star's surface is favoured for the outflow. First, a stellar wind with the mass loss rate we derive here would inevitably produce some blueshift in all the lines produced in the vicinity of the star, which is not observed. Second, this mass loss rate is rather close to the upper limit found by Beust et al. (2001) and it would affect the FEBs signatures, which is again not observed. A flow coming from regions above the disk midplane does not suffer from these problems.

In both cases, what causes the outflow is nevertheless not quite clear. The temperature we assumed is far from being high enough to generate a thermally driven wind. The thermal speed, 


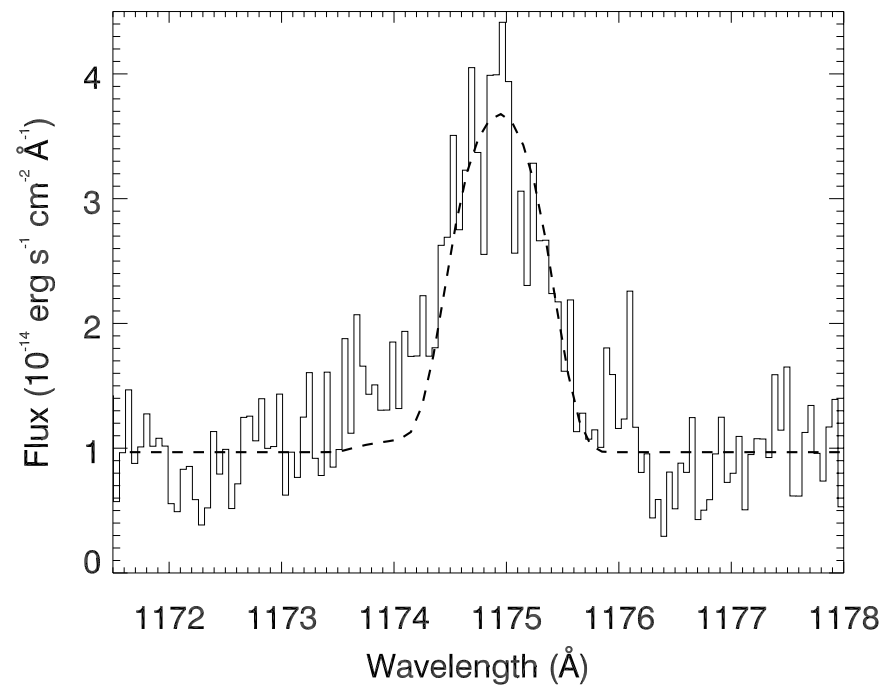

Fig. 7. C III excited line in the Lif 2a spectrum (full line) compared to the synthetic profile (dashed line) obtained with the disk-wind model discussed in Sect. 5.2.

$v_{\mathrm{th}}=26 \mathrm{~km} \mathrm{~s}^{-1}$, is indeed much smaller than the observed outflow velocity, which is $200 \mathrm{~km} \mathrm{~s}^{-1}$. Similarly, $\beta$ Pictoris is surely not bright and hot enough for its radiation pressure to drive the outflow. Most likely, a complex combination of several mechanisms is implied and we did not intend to thoroughly investigate the physics leading to an outflow as suggested by the present modeling. We defer to a future work a detailed examination of the various mechanisms that may be involved in driving, heating and collimating the outflow.

\section{Discussion}

\subsection{Model prediction and other chromospheric diagnostics}

The principal issue of this paper is to investigate the relevance of a chromospheric model to explain the emission spectrum of $\beta$ Pictoris, newly revealed by FUSE. The model we propose is able to reproduce not only the FUV emission features but also absorption lines of Mg II and C IV, spanning a large domain of wavelengths, in the near-UV.

In addition, when calculating the $\mathrm{H} \alpha$ line profile, we find no differences between our synthetic profile and what is produced by the Kurucz model of $\beta$ Pictoris, once convolved with the stellar $v \sin i$ (see Fig. 8). This comes from the fact that our model, even though it includes high temperature regions above the photosphere, is much too optically thin to have visible effect on the $\mathrm{H} \alpha$ profile. We found that the optical depth ratio between Ly $\alpha$ and $\mathrm{H} \alpha$ is about $10^{6}$, for the model discussed here.

Additional modeling of other lines probing different temperature regimes is necessary to achieve a better description of the temperature run above the photosphere. Observing the $\mathrm{N} \mathrm{V}$ resonance doublet (1238-1240 ̊) or Ly $\alpha$ would be most helpful, in this context. The former line would help constraining the temperature law bewteen the C IV and O VI formation regions. Likewise, Ly $\alpha$ which is known to be an excellent diagnostic for chromospheric regions, would provide reliable information

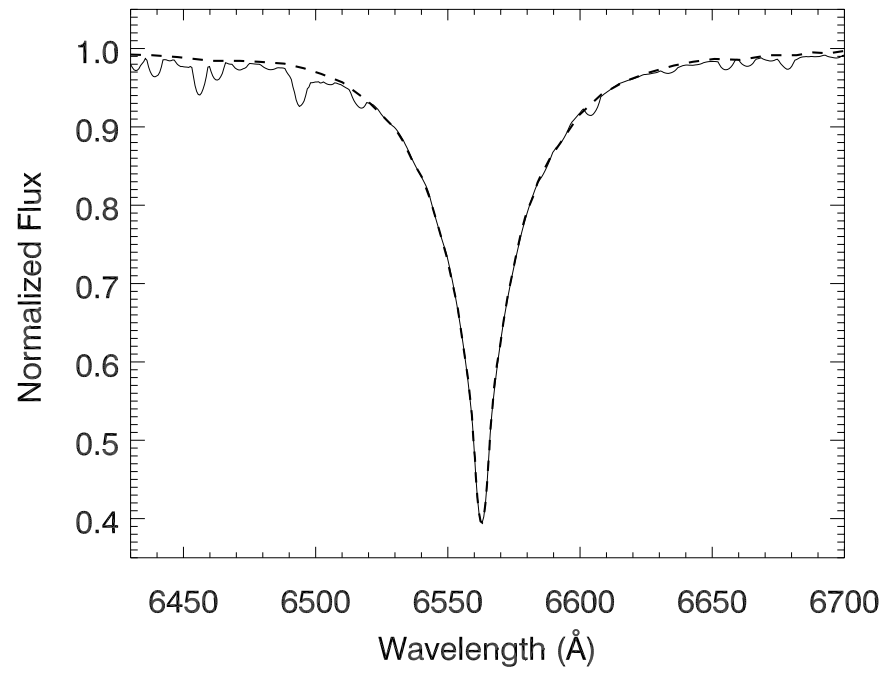

Fig. 8. H $\alpha$ : Comparison between profiles resulting from Kurucz photospheric model (full line) and our chromospheric model (dashed line).

on the temperature regime from $10000 \mathrm{~K}$ to $25000 \mathrm{~K}$. Besides these lines, C II resonance line at $1335 \AA$ should also be observed in emission, if the model is correct.

The model predicts that $\operatorname{Ly} \alpha$ should intrinsically appear as a single peak, broad emission feature. When taken into account, the contamination of this intrinsic profile by the interstellar and circumstellar Ly $\alpha$ absorptions will change it into a double-peaked emission profile. Fitting the observed profile would not only provide information on the temperature law in the deep chromosphere but also allow us to precisely derive the $\mathrm{HI}$ column density and the micro-turbulent velocity $b_{\mathrm{HI}}$. In any case, the changes deduced from Ly $\alpha$ fitting must be small enough that they do not change the fits already obtained in the present work. Note that because of the interstellar contamination, meaningful comparisons between observed and synthetic Ly $\alpha$ profiles will be restricted to the external wings of the emission component.

\subsection{Measure and origin of the chromospheric activity}

The ability of our model to explain the properties of the emission lines in $\beta$ Pictoris spectrum confirms that this star is undergoing a chromospheric active phase. Although surprising for a star of this spectral type, this behaviour has been observed already in more evolved A stars of later spectral type, like $\alpha$ Aql (Altair) or $\alpha$ Cep (Freire-Ferrero et al. 1995; Simon \& Landsman 1997; Gouttebroze et al. 1999; Redfield et al. 2002).

$\beta$ Pictoris is most likely the earliest $\mathrm{A} \mathrm{V}$ star for which chromospheric emission has been observed so far, since the evidence for such an emission in $\tau^{3}$ Eri is only marginal and would require much deeper exposure for confirmation (Simon \& Landsman 1997). At best, the sample of known mainsequence mid A-type stars exhibiting conspicuous signs of chromospheric activity is therefore made of two stars. On the opposite, a large number of younger objects of same spectral type, like the Herbig Ae stars, do present spectral signatures of a strong activity. Assuming that a unique mechanism drives 
the observed activity of A-type stars, from the PMS phase to the ZAMS, this suggests that the efficiency of this mechanism is decaying with time. In other words, if observed at younger age, $\beta$ Pictoris would exhibit more activity and vice versa. We might well be witnessing the final stage of the active phase of a mid A V-type star.

Whatever the mechanism sustaining activity, the existence of an atmospheric structure including a chromosphere-TR complex implies that a large amount of non radiative energy is dissipated above the photosphere of $\beta$ Pictoris. We can estimate it by the calculation of the radiative losses, i.e., the amount of energy radiated by the lines and continua that are formed in the chromosphere and transition region. We recall here that the radiative losses are defined by:

$E^{-}(r)=4 \pi \int_{0}^{\infty}\left(\epsilon_{v}-\chi_{v} J_{v}\right) \mathrm{d} v \quad\left(\operatorname{ergs~cm}{ }^{-3} \mathrm{~s}^{-1}\right)$.

This equation expresses the total amount of emission minus the total amount of absorption ( $\epsilon_{v}$ is the gas emissivity, $\chi_{v}$ is the absorption coefficient and $J_{v}$ is the mean intensity of radiation). $E^{-}(r)$ is then positive when the medium cools by radiation. The sum of the contributions of a large number of radiators and NLTE effects have to be taken into account in the calculation of $E^{-}(r)$. The present work being limited to a few elements, we can only estimate lower limits on the radiative losses $E^{-}(r)$.

In order to calculate $E^{-}(r)$, we need to solve the radiative transfer equation, that will provide us with the mean radiation intensity as well as with the source function of the transitions we are dealing with. The lines that have been used in this work provide information on the gas physical conditions throughout the chromosphere and TR, though parts of these regions are poorly constrained. Therefore, our calculations of the radiative losses only provide a rough estimate of the energy required to sustain a chromosphere-TR complex in $\beta$ Pictoris close environment. $E_{\text {tot }}^{-}$was calculated by integrating the radiative losses $E^{-}(r)$ between the two points $r_{1}$ and $r_{2}$ where $T=T_{\min }$ and $T=T_{\max }$ respectively:

$E_{\text {tot }}^{-}=\int_{r_{1}}^{r_{2}} 4 \pi r^{2} E^{-}(r) \mathrm{d} r \quad\left(\operatorname{ergs~s}^{-1}\right)$.

For the adopted model, the values we have found are listed in Table 4.

The ratio of the radiative losses to the bolometric luminosity of the star is $R=2.3 \times 10^{-6}$. Using the Barnes \& Evans (1976) relation, Simon \& Landsman (1997) converted the apparent fluxes measured for a few chromospheric emission lines into stellar surface fluxes for $\alpha \mathrm{Aql}$ and $\alpha$ Cep. In terms of these chromospheric/TR fluxes, $\beta$ Pictoris is slightly more active (by a factor of 4-6) than these stars.

Using Eqs. (5) and (6) for more than 20 transitions (lines and continua), Bouret \& Catala (1998) showed that for Herbig A0e stars, which are believed to be the progenitors of mainsequence A stars, the radiative losses in the chromosphere reach up to a few $10^{-2}$ of the stellar bolometric luminosity. These stars have significant stellar winds ( $\dot{M}$ up to a few $\left.10^{-8} M_{\odot} \mathrm{yr}^{-1}\right)$ and extended chromosphere of moderate temperatures $(T \sim 20000 \mathrm{~K})$. The dominant contributors to their radiative losses are the $\mathrm{H} \alpha$ line and the Balmer continuum,
Table 4. Contributions to the radiative losses from the various lines formed in the outer atmosphere of $\beta$ Pictoris.

\begin{tabular}{|c|c|}
\hline Transitions & $E_{\text {tot }}^{-}\left(\operatorname{ergs~s}^{-1}\right)$ \\
\hline C II.......................... & $2.2 \times 10^{+26}$ \\
\hline 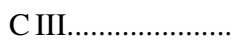 & $8.4 \times 10^{+27}$ \\
\hline C III* ...................... & $9.8 \times 10^{+26}$ \\
\hline 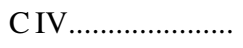 & $2.4 \times 10^{+28}$ \\
\hline 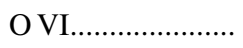 & $3.6 \times 10^{+27}$ \\
\hline C III Cont............. & $2.6 \times 10^{+28}$ \\
\hline C IV Cont.............. & $1.4 \times 10^{+28}$ \\
\hline O VI Cont.............. & $2.2 \times 10^{+28}$ \\
\hline Total..... & $9.9 \times 10^{+28}$ \\
\hline
\end{tabular}

while the other major lines and continua (Magnesium and Carbon) produce negligible contributions (Bouret \& Catala 1998). In the case of the prototypical Herbig Ae star AB Aur, the highest temperatures are reached in discrete regions produced by shocks in the wind; in these regions the resonance lines of C IV and N V dominate the radiative losses. Obviously, the $\beta$ Pictoris case is very different for four reasons: (1) the lower density in the the chromosphere/TR makes the radiative losses lower - (2) The physical extent of the emitting regions (chromosphere/TR) is much smaller in $\beta$ Pictoris than in Herbig Ae stars; as a comparison, AB Aur chromosphere extends over about 1.6 stellar radii while $\beta$ Pictoris chromosphere/TR is only about $0.4 R_{\text {ph }}$ thick - (3) much higher temperatures are reached in the $\beta$ Pictoris chromosphere/TR (4) the chromosphere/TR is not discrete but rather, spherically symmetric. Therefore, a direct comparison to Herbig Ae stars is difficult, but we found that $\beta$ Pictoris is much less active than these PMS objects.

In the $\alpha \mathrm{Aql}$ and $\alpha$ Cep case, as well as in many Herbig Ae/Be stars, X-ray emission has been detected (Schmitt et al. 1985; Zinnecker \& Preibisch 1994). $\alpha$ Aql being, from many points of view, similar to $\beta$ Pictoris, some X-ray emission should arise in the latter. A simple scaling to the activity level measured for $\alpha$ Aql gives $\log L_{\mathrm{X}} / L_{\mathrm{bol}} \approx-7$ for $\beta$ Pictoris. This is roughly two orders of magnitude smaller than what is measured for active solar-type stars (Mathioudakis et al. 1995) and about one order of magnitude smaller than typical values for early F-type stars like Procyon (see Drake et al. 1995). In the latter cases, the corona is heated up to $\log T_{\mathrm{e}} \approx 6.0-6.2$. As far as $\alpha$ Aql is concerned, a thourough analysis of the various causes leading to a low emission measure lead Freire-Ferrero et al. (1995) to suggest that a warm corona is present close to the star, with $\log T_{\mathrm{e}} \approx 5.7-5.8$. If the analogy with $\alpha$ Aql is valid, a warm corona is therefore expected for $\beta$ Pictoris, though it cannot be firmly predicted on the basis of the present dataset. Consequently, the star should have an intrinsic X-ray emission, originating in this corona. To our knowledge, no such detection has been claimed for $\beta$ Pictoris, which most likely results from the presence of a large quantity of absorbing gas in the soft X-ray range around $\beta$ Pictoris.

The nature of the source of energy that heats the gas is still far from being determined. Should the solar analogy prevail, 
the gas heating would be expected to be caused by magnetic and/or accoustic energy dissipation. This, in turn, implies that a sub-photospheric convection zone exist in $\beta$ Pictoris. Although in contradiction with the standard theory of stellar evolution, this conclusion is supported by several theoretical works showing that such sub-photospheric convective regions could exist even in early A-type stars (Zahn 1980; Bohn 1984; Zahn 1993).

Alternatively, the ultimate origin of this non-radiative energy may be stellar rotation, through the transformation of rotational energy into turbulent energy in the star's interior, through shear instabilities (Lignières et al. 1996). This model, first developed for Herbig Ae/Be stars, assumes that such an energy transformation is caused by the violent rotational braking that the wind exerts on the star's surface. The stronger the wind, the higher the amount of energy that can be extracted. In $\beta$ Pictoris case, adopting $\dot{M}=2.5 \times 10^{-14} M_{\odot} \mathrm{yr}^{-1}$ (see Sect. 3.2), we found that this mechanism provides an energy reservoir lower than the radiative losses by about two orders of magnitude: $E_{\text {shear }}^{-} / E_{\text {tot }}^{-} \propto 5 \times 10^{-2}$. However, this model does not take into account the effect of a magnetic field that should be produced by a dynamo mechanism in the subphotospheric turbulent layers. In that case, the amount of energy that could be extracted from the turbulent layer is likely to be larger by two to three orders of magnitudes (Lignières et al. 1996). This promising approach needs to be completed by its inclusion in a self consistent model of the coupling of the magnetic field production with the turbulent subphotospheric layers and may reconcile the energy production rate with our estimates of the radiative losses. This scenario is especially interesting for our case since our modeling work has required the introduction of a superrotational broadening to achieve a good fit of the emission lines which suggests that rotation is indeed an important component of the physics of the chromosphere-TR. Because of the high level of differential rotation between the chromosphereTR and the photosphere, the torque exerted by the weak wind of $\beta$ Pictoris may efficiently induce subphotospheric turbulence.

Regardless of the nature of this energy source, the heating is apparently effective on a very thin region above the stellar disk, even when the presence of a potential corona is taken into account. We already showed that $\beta$ Pictoris chromosphere/TR complex is much thinner than those of Herbig Ae stars. On the other hand, it is very similar to the physical size inferred for $\alpha$ Aql and $\alpha$ Cep by Gouttebroze et al. (1999). This may indicate that the efficiency of the mechanism generating the magnetic field is decaying with time and would explain why mainsequence A-type stars lack a strong magnetic field. In cooler stars, the strong magnetic fields trap and heat the gas in closed loop structures and the energy deposition is weaker at the base of the magnetic structure. Consequently, the chromosphere and transition region are even thinner than in $\beta$ Pictoris (a few percent of stellar radius for the Sun) but their coronae can extend over more than one stellar radius.

\section{Conclusions}

Although unexpected for a mid A V type star like $\beta$ Pictoris the emission lines revealed by FUSE are most likely a clue for the existence of a significant stellar activity. We have shown that not only these emission lines but also several absorption lines can be reproduced with a simple model, which involves a chromosphere and a transition region. This theoretical framework is new for $\beta$ Pictoris and is most helpful for interpreting the aforementioned observed characteristics of the star, which could not be explained in the FEBs framework.

The shape and intensity of C III and O VI emission lines have been used to place constraints on the temperature law and size of the chromosphere and transition region. The latter were found such that their presence does not affect purely photospheric profiles, like $\mathrm{Mg}$ II $\mathrm{h}$ and $\mathrm{k}$ or the $\mathrm{H} \alpha$ line for instance. The model predicts that the Ly $\alpha$ line should appear as a rather strong intrinsic emission line, on top of which an absorption trough is formed by the IS hydrogen. Fitting the observations with the theoretical line will provide a direct and accurate determination of the hydrogen column density on the line of sight to $\beta$ Pictoris; this however requires HST/STIS observations yet to come.

The puzzling blueshift of the C III* line profile has been successfully interpreted in terms of a thin warm wind coming from the massive CS disk of $\beta$ Pictoris. Although we did not develop a general model of this region, the prescriptions we used strongly suggest that the gas is flowing with a quite small angle with respect to the disk plane. This conclusion may relate the $\beta$ Pictoris case to that of a few Herbig Ae stars showing the same general behaviour, though observed through very different ionization conditions.

Nevertheless, several question remain open, mostly on the theoretical side. First of all, the origin and nature of the process(es) leading to the energy deposition responsible for the gas heating in the outer atmosphere of $\beta$ Pictoris is still to be clearly identified.

Second, the mechanisms driving and heating the disk wind are unknown as well, although a combination of both thermal and radiative pressure might be efficient enough to explain the calculated mass loss rate and observed blueshift.

Finally, assuming that a unique mechanism drives the observed activity of A-type stars from the PMS stage to the ZAMS phase, our results suggest that the effectiveness of this mechanism decays with time, likewise the decay of activity during the main-sequence lifetime of solar-type stars. We might be witnessing here the final active stage of a mid A V star. It is therefore of importance to increase the sample of A stars showing active signature, for different evolutionnary stages.

Acknowledgements. We thank Anne-Marie Lagrange and Hervé Beust for fruitful discussions about the FEBs model. We also wish to warmly thank Andrea Dupree, Michelle Eidelsberg, Rubens FreireFerrero and Pierre Gouttebroze for interesting comments on the stellar activity and molecular data of CO. Finally, we acknowledge Thomas Ayres for kindly communicating his lines irradiance measurements to us. This work is based on data obtained for the Guaranteed Time Team by the NASA-CNES-CSA FUSE mission operated by the Johns Hopkins University. Financial support to U. S. participants has been provided by NASA contract NAS5-32985. 


\section{References}

Babel, J. 1995, A\&A, 301, 823

Barnes, T. G., \& Evans, D. S. 1976, MNRAS, 174, 489

Barrado y Navascues, D., Stauffer, J. R., Song, I., \& Caillault, J.-P. 1999, ApJ, 520, L123

Bathia, A., \& Kastner, S. O. 1993, ApJ, 408, 744

Beust, H., \& Tagger, M. 1993, Icarus, 106, 42

Beust, H., \& Morbidelli, A. 2000, Icarus, 143, 170

Beust, H., Karmann, C., \& Lagrange, A. M. 2001, A\&A, 366, 945

Bohn, H. U. 1984, A\&A, 136, 338

Bouret, J.-C., \& Catala, C. 1998, A\&A, 340, 163

Bouret, J.-C., \& Catala, C. 2000, A\&A, 359, 1011

Bruhweiler, F. C., Grady, C., \& Kondo, Y. 1991, ApJ, 371, L27

Calvet, N., Hartmann, L., \& Kenyon, S. J. 1993, ApJ, 402, 623

Cardelli, J., Clayton, G., \& Mathis, J. 1989, ApJ, 345, 245

Deleuil, M., Gry, C., Lagrange-Henri, A. M., et al. 1993, A\&A, 267, 187

Deleuil, M., Bouret, J.-C., Roberge, A., et al. 2001, ApJ, 557, L67

Drake, J. J., Laming, J. M., \& Widing, K. G. 1995, ApJ, 443, 393

Eidelsberg, M., Benayoun, J. J., Viala, Y., \& Rostas, F. 1991, A\&AS, 90, 231

Freire Ferrero, R., Gouttebroze, P., Catalano, S., et al. 1995, ApJ, 493, 1011

Goldreich, P., \& Ward, W. R. 1973, ApJ, 183, 1051

Gouttebroze, P., Freire Ferrero, R., Marilli, E., \& Catalano, S. 1999, A\&A, 348, 198

Hartmann, L., \& Calvet, N. 1995, AJ, 109, 1846

Hobbs, L. M., Welty, D. E., Lagrange-Henry, A. M., et al. 1988, ApJ, 334, L41

Hubeny, I. 1985, in Progress in Spectral Line Formation Theory, ed. J. E. Beckmann, \& L. Crivellari (Reidel, Dordrecht)
Kondo, Y., \& Bruhweiler, F. C. 1985, ApJ, 291, L1

Lagrange-Henri, A. M., Vidal-Madjar, A., \& Ferlet, R. 1988, A\&A, 190,2775

Lanz, T., \& Catala, C. 1992, A\&A, 257, 663

Lanz, T., Heap, S., \& Hubeny, I. 1995, ApJ, 447, L41

Lecavelier des Etangs, A., Vidal-Madjar, A., Roberge, A., et al. 2001, Nature, 412, 706

Lignières, F., Catala, C., \& Mangeney, A. 1996, A\&A, 314, 465

Mathioudakis, M., Fruscione, A., Drake, J. J., et al. 1995, A\&A, 300, 775

Mihalas, D., \& Kunasz, P. 1978, ApJ, 219, 635

Mihalas, D. 1978, in Stellar Atmospheres, $2^{\text {nd }}$ edition

Redfield, S., Linsky, J. L., \& Dupree, A. K. 2002, AAS, 199, 1312

Roberge, A., Feldman, P. D., Lagrange, A. M., et al. 2000, ApJ, 538, 904

Schmitt, J. H. M. M., Golub, L., Harnden, F. R., et al. 1985, ApJ, 290, 307

Simon, T., \& Landsman, W. 1997, ApJ, 483, 435

Simon, T., \& Ayres, T. 1998, ApJ, 500, L37

Smith, B., \& Terrile, R. 1984, Science, 226, 1421

Tout, C., \& Pringle, J. E. 1995, MNRAS, 272, 528

Vidal-Madjar, A., et al. 1994, A\&A, 290, 245

Vidal-Madjar, A., Lecavelier des Etangs, A., \& Ferlet, R. 1998, Planet. Space Sci., 46, 629

Viera, S. L. A., Pogodin, M. A., \& Franco, G. A. P. 1999, A\&A, 345, 559

Vigneron, C., Mangeney, A., Catala, C., \& Schaztman, E. 1990, Solar Phys., 128, 287

Zahn, J. P. 1980, in Proc. IAU Colloq. 51, Stellar turbulence, 1

Zahn, J. P. 1993, ASP Conf. Ser. 40: IAU Colloquium 137, Inside the stars, 236

Zinnecker, H., \& Preibisch, T. 1994, A\&A, 292, 152 